\title{
RELAÇÃO DO PICO DE FLUXO EXPIRATÓRIO COM O TEMPO DE FONAÇÃO EM PACIENTES ASMÁTICOS
}

\author{
Relation of peak-flow with fonation time in asmatic patients
}

\author{
Débora Cardoso Rossi ${ }^{(1)}$, Danielle Ferreira Munhoz ${ }^{(2)}$, Cristiano Rabelo Nogueira ${ }^{(3)}$, Teresa \\ Cristina Moura de Oliveira ${ }^{(4)}$, Ana Teresa Brandão de Oliveira Britto ${ }^{(5)}$
}

\begin{abstract}
RESUMO
Objetivo: investigar a possível relação do Pico de Fluxo Expiratório (PFE) com o Tempo Máximo de Fonação (TMF) em pacientes asmáticos. Métodos: a pesquisa enfocou 16 indivíduos com o diagnóstico de asma pura, sendo 9 do gênero feminino e 7 do gênero masculino, atendidos no serviço ambulatorial de pneumologia do Hospital Júlia Kubitschek em BH. A equipe de pneumologia diagnosticou, classificou a asma e realizou o teste PFE. Posteriormente os pacientes foram submetidos à extração de tempo Máximo de Fonação (TMF) para os sons /a/, /i/, /u/, /e áfono/, /e sonoro/, /s/ e /z/. Resultados: dos pacientes atendidos havia 12,50\% (2) com asma tratada, 18,75\% (3) com asma persistente leve, $43,75 \%$ (7) com asma persistente moderada, 6,25\% (1) com asma moderada grave e $18,75 \%$ (3) com asma persistente grave. Foi observado que quando o TMF encontrava-se reduzido ocorria também uma redução do PFE e quando havia um aumento do TMF era encontrado no PFE valor também maior. Conclusão: foi possível observar uma associação e uma correlação entre as medidas TMF e PFE que sugerem uma relação direta dessas medidas.
\end{abstract}

DESCRITORES: Pico do Fluxo Expiratório; Asma; Fonoaudiologia; Doença Pulmonar (Especialidade)

\section{INTRODUÇÃO}

Os distúrbios vocais são caracterizados por desvios que comprometem a inteligibilidade e a efetividade da comunicação oral, sejam eles manifestados por meio de alterações na freqüência, intensidade e/ou na qualidade vocal, por transtornos no funcionamento laríngeo, respiratório e/ou do trato vocal ${ }^{1}$.

O sistema respiratório funciona como ativador da voz, portanto qualquer comprometimento da função aérea pode exercer um efeito direto sobre a fala e a

(1) Fonoaudióloga da Clinica FONO de Belo Horizonte; Especialista em Voz e Motricidade Oral.

(2) Fonoaudióloga; Especialista em Voz.

(3) Médico do Hospital Beneficência Portuguesa de São Paulo; Doutorando em Pneumologia pela Universidade Federal de São Paulo.

(4) Fonoaudióloga, Professora do Centro Universitário Izabela Hendrix de Minas Gerais, Coordenadora do Centro de Especialização em Fonoaudiologia Clínica de Belo Horizonte; Mestre em Fonoaudiologia pela Pontifícia Universidade Católica de São Paulo.

(5) Fonoaudióloga Coordenadora do curso de Fonoaudiologia da Pontifícia Universidade Católica de Minas Gerais; Doutoranda em Lingüística pela Pontifícia Universidade Católica de Minas Gerais. voz (intensidade, altura e qualidade) ${ }^{2}$. Doenças pulmonares afetam diretamente a fisiologia respiratória, comprometendo o fluxo aéreo. Uma vez que a fisiologia respiratória está intimamente relacionada com a fisiologia vocal, medidas pneumofônicas podem detectar alterações do fluxo aéreo expiratório. A esses indivíduos é indicada a avaliação pneumológica.

A medida do tempo máximo de fonação (TMF) é descrita amplamente na literatura científica com grande ênfase à detecção de alterações em nível glótico, sendo escasso os relatos sobre alterações em nível pulmonar. Como a função pulmonar está diretamente relacionada com a produção da voz é de se esperar que o TMF possa estar alterado, também, em indivíduos com doenças pulmonares ${ }^{3}$.

A medição do TMF é o parâmetro utilizado para obtenção de medidas pneumofônicas. O valor da medição do TMF é obtido através de sustentação vocal do som da fala vozeado ou não vozeado de forma prolongada em uma só expiração ${ }^{4}$.

A capacidade vital pulmonar (CV), volume medido na boca entre as posições de inspiração plena e expiração completa, representa o maior volume de ar mobilizado. ACV varia enormemente entre indivíduos, resultando de forma indireta em grande variação no TMF. 
O TMF nunca corresponderá a $100 \%$ da CV, pois uma pequena porção dessa CV será perdida no início da manobra antes de iniciar a fonação, devido à grande pressão alveolar e pela aproximação laríngea incompleta ${ }^{5}$. Acredita-se que a CV juntamente com a pressão alveolar e a válvula laríngea para o fluxo aéreo, sejam fatores chaves que regulam o TMF. A variação do TMF, que não pode ser justificada pela CV, é presumidamente atribuída à resistência laríngea e ao fluxo aéreo ${ }^{6,7}$.

A avaliação vocal diagnóstica, com medidas de fluxo na proporção s/z (/s/ som surdo e /z/ som sonoro), entre 0,8 e 1,2 é esperado para indivíduos normais. Valores baixos são indicativos de hiperadução das pregas vocais e valores acima de 1,2 revelam falta de coaptação glótica. A proporção dos tempos de emissão do /e/ áfono e /e/ sonoro é uma manobra para identificação de componente hipercinético. A medida isolada da vogal áfona /e/ pode clinicamente apontar como o paciente controla a saída progressiva do ar por meio de seu suporte respiratório, sugerindo deficiências no mecanismo respiratório diante de tempos curtos de emissão. Medidas de normalidade giram em torno de 16 a 18 segundos ${ }^{4}$.

O teste de vogais sustentadas para a avaliação de voz é uma das medidas tradicionais para a investigação da fonação. Indica a habilidade do paciente em controlar as forças aerodinâmicas da corrente pulmonar e as forças mioelásticas da laringe. Por excelência, é um teste de eficiência glótica. É solicitado ao paciente, após inspiração profunda, que emita cada vogal num tempo o mais prolongado possível. O tom e a intensidade usados devem ser habituais, indicando naturalidade. Se o paciente utilizar o ar de reserva, tal fato deverá ser registrado, mas nenhuma interferência ou sugestão deve ser feita no sentido de evitar que ele haja dessa forma, uma vez que o objetivo é observar de que forma ele usa a quantidade de ar disponível ${ }^{3,4,8,9}$.

Para falantes do português do Brasil (experiência com falantes da cidade de São Paulo) considera-se uma média de 20 segundos (s) para os homens e 14s para as mulheres; para as crianças, até a puberdade, esses valores acompanham a idade em anos relacionando-se 1s para cada ano de vida (uma criança, com 3 anos, sustenta uma vogal por 3s).Tempo máximo de fonação abaixo de 10s para indivíduos adultos é indicativo de comprometimento vocal significativo ${ }^{9,10}$.

A medição do tempo fonatório requer apenas o cronômetro e a observação clínica, sendo um teste simples e comum na prática fonoaudiológica. Caso exista correlação entre o TMF e o Pico de Fluxo Expiratório (PFE) poderia, também, ser utilizado como triagem de alteração do fluxo respiratório no exame fonoaudiológico de rotina.

Esta pesquisa tomou como base a medida do tempo fonatório em pacientes asmáticos permitindo a avaliação de vários graus de acometimento broncopulmonar com as variações do TMF e do pico de fluxo expiratório (PFE), sendo que esta última foi obtida através de valores objetivos para comparação.

Estima-se que $4 \%$ dos americanos sofrem de asma e que $7 \%$ da população apresentarão essa desordem em algum momento ${ }^{11}$. A asma é conceituada como distúrbio ventilatório que se caracteriza pelo aumento de reatividade da traquéia e brônquios que resulta no estreitamento das vias aéreas, promovendo obstrução ao fluxo aéreo (aumento da resistência) e comprometendo a capacidade respiratória (inspiração e expiração). O aumento da resistência ao fluxo aéreo nas vias aéreas pulmonares, que é maior para a expiração que para a inspiração, pode ser causado por maior produção de muco, por espasmo da musculatura brônquica ou por espessamento da parede das vias aéreas ${ }^{11}$. É, portanto, uma doença inflamatória crônica que se caracteriza por uma limitação ao fluxo aéreo.

A asma pode ser classificada de acordo com sua gravidade:

Intermitente - apresenta sintomas menores ou iguais a uma vez por semana, atividade normal, crise ocasional e sintoma noturno raro;

Persistente leve - apresenta sintomas maiores ou iguais a uma vez por semana e menor que uma vez/dia, atividade com diminuição de limitação, crises menos freqüentes e sintomas noturnos ocasionais;

Persistente moderada - apresenta sintomas diários, atividade com aumento de limitação, crises freqüentes, sintomas noturnos comuns e, por fim, a asma

Persistente Grave - que possui sintomas diários, atividade com limitação diária, crises muito freqüentes e sintomas noturnos diários ${ }^{12}$.

O propósito principal da ventilação é a troca gasosa e, secundariamente, a fala. O padrão respiratório mais eficiente para a troca gasosa é diferente daquele utilizado para a fala. Numa crise asmática há mais dificuldade para a fala pela própria prioridade para a troca gasosa ${ }^{13}$.

Os asmáticos inspiram maior volume de ar do que um indivíduo normal e expiram volume similar. Entretanto, eles usam menor proporção desse volume para a fala, sendo que este último se reduz à medida que a asma se torna mais severa ${ }^{13}$. O aumento do volume pulmonar no asmático resulta em aumento do diâmetro das vias aéreas, aumentando ainda a contração pulmonar passiva expiratória, compensando o aumento da resistência à expiração, que é maior do que para a inspiração.

De acordo com um estudo realizado em 14 pacientes com asma e 10 indivíduos normais, o volume expirado durante a fala foi menor nos asmáticos. Reciprocamente, o volume expirado na fase em que 
não produziu voz foi em média 2 a 4 vezes maior nos asmáticos. Os asmáticos tiveram fluxo inspiratório mais lento e fluxo expiratório mais rápido e, na fala, tiveram tempo mais curto para o ciclo respiratório total ${ }^{13}$. Indivíduos saudáveis utilizam maior média de volume para a fala.

Os asmáticos apresentam somente dificuldade expiratória e produzem menos sílabas por respiração (ciclo respiratório mais curto) ${ }^{11}$. A fala nos asmáticos difere dos saudáveis e a observação desse estudo mostra que não há diferença na microestrutura - como para os segmentos de sons da fala ou para sílabas. A diferença é observada na macroestrutura de prosódia e do ritmo da fala e da respiração. O menor tempo usado pelo asmático para fala denota sua necessidade aumentada para produzir a troca metabólica ${ }^{11}$.

A clínica fonoaudiológica da voz depara-se com algumas medições da clínica pneumológica em relação à capacidade respiratória (sobretudo a capacidade expiratória). Dentre elas encontra-se o pico de fluxo expiratório. O Pico de Fluxo Expiratório (PFE) é um parâmetro espirométrico com utilização de apareIhos portáteis, de baixo custo, independente de fonte elétrica e está diretamente relacionado com a força muscular e volume pulmonar. O PFE é indicado para monitorização, a curto e a longo prazo, das doenças pulmonares obstrutivas, especialmente a asma brônquica. O PFE baseia-se na medida do sopro expiratório ${ }^{6,14,15}$ É freqüentemente realizado na clínica pneumológica como procedimento de rotina.

Esta pesquisa foi baseada em questões pertinentes que envolveram a contribuição do pneumologista para a clínica fonoaudiológica da voz; comparação do PFE com o TMF, que serão discutidos e apresentados neste trabalho.

\section{MÉTODOS}

Inicialmente foram analisados 30 prontuários referentes aos indivíduos atendidos no serviço ambulatorial de pneumologia do Hospital Júlia Kubitschek (HJK) em Belo Horizonte.

Foram incluídos indivíduos com diagnóstico de asma, entre 20 e 60 anos. Os critérios de exclusão foram indivíduos menores de 20 anos (devido às alterações vocais da adolescência) e maiores de 60 anos (devido às alterações vocais do envelhecimento). Observou-se a presença de arqueamento de pregas vocais sem patologia laríngea em indivíduos acima de 60 anos. Com o envelhecimento ocorrem modificações das camadas superficial e intermediária, alterações na vibração da mucosa e atrofia da musculatura ${ }^{16}$. Também foram excluídos tabagistas há mais de 10 anos e pacientes com qualquer outra patologia associada e que pudesse alterar a função pulmonar e a voz.

Do total, 14 foram excluídos por apresentarem outra patologia associada à asma.
Este estudo enfocou 16 indivíduos com o diagnóstico de asma pura, sendo nove pacientes do gênero feminino e sete do gênero masculino, entre $30 \mathrm{e}$ 60 anos de idade. Os dados foram coletados durante os meses de agosto a setembro do ano de 2004.

Os indivíduos foram classificados pelo grau da asma.

A partir de sua chegada ao Serviço Ambulatorial de Pneumologia do Hospital Júlia Kubitschek, em Belo Horizonte, os pacientes foram avaliados pela equipe de pneumologia que diagnosticou, classificou a asma e realizou teste de PFE. Posteriormente os pacientes foram encaminhados à avaliação fonoaudiológica dentro do próprio ambulatório de pneumologia.

A avaliação fonoaudiológica constou da emissão dos fones [a], [i] e [u] sonoros; [e] áfono e [e] sonoro, [s] e [z] e a medida da relação s/z $\mathbf{z}^{4,8-10,17}$. A avaliação fonoaudiológica não ultrapassou os 20 minutos. Os pacientes permaneceram sentados, adequadamente posturados, em ambiente sem ruídos. A medição foi feita conjuntamente por duas fonoaudiólogas (autoras) sendo que uma ficou responsável pela parte técnica (cronômetro e gravação em fita cassete) e a outra pelas anotações específicas contidas no protocolo de avaliação.

Foram selecionadas as vogais /a/, li/, / $/$ / para esse teste. $\mathrm{O} / \mathrm{a} /$ é uma vogal oral, central, aberta, que por sua natureza articulatória torna evidente as mínimas alterações no equilíbrio mioelástico da laringe; é a vogal que mais evidencia a disfonia e, portanto, a vogal de eleição para qualquer teste de voz. A vogal /i/é a mais aguda do português e a mais fechada anteriormente, enquanto a /u/ é a mais grave e a mais fechada, posteriormente.

A proporção dos tempos de emissão do /e/ áfono e /e/ sonoro é uma manobra para identificação de componente hipercinético. A medida isolada da vogal áfona /e/ pode clinicamente apontar como o paciente controla a saída progressiva do ar por meio de seu suporte respiratório, sugerindo deficiências no mecanismo respiratório diante de tempos de emissão curtos. Medidas de normalidade giram em torno de 16 a 18 segundos ${ }^{4}$.

Para as medidas das fricativas mediais, surda e sonora assume-se o pressuposto de que, numa fonação sustentada, um indivíduo com dinâmica fonatória normal é capaz de utilizar a suplência de ar pulmonar de modo eficiente, o que não acontece com os pacientes disfônicos.

Quando se emite a fricativa medial surda/s/, após uma inspiração profunda e de forma prolongada, avalia-se o suporte aéreo pulmonar, principalmente quanto à habilidade de controlá-lo, já que não há vibração de pregas vocais nesse som; portanto, a medida de / $\mathrm{s} /$ nos oferece a avaliação da fonte friccional do som. Quando se emite a consoante sonora medial/z/, tam- 
bém após inspiração profunda de forma prolongada, acopla-se à fonte friccional inicial a fonte glótica, e pode-se observar o comportamento vocal resultante.

As medidas isoladas dos tempos máximos do fonema fricativo medial surdo $/ \mathrm{s} / \mathrm{e}$ do fonema fricativo medial sonoro /z/ não têm valor clínico, mas a relação entre essas duas medidas, a chamada relação s/z é um valioso recurso para avaliação da dinâmica fonatória e da competência glótica. Espera-se que os valores sejam os mesmos ou com pequena variação entre o fonema surdo e o fonema sonoro, uma vez que a dinâmica fonatória normal é capaz de utilizar a suplência de ar pulmonar de maneira eficiente. Uma relação s/z entre 0,8 e 1,2 é esperada para indivíduos normais. Valores baixos são indicativos de hiperadução das pregas vocais e valores acima de 1,2 revelam falta de coaptação glótica ${ }^{2,4,8-10}$.

Na presente pesquisa foi utilizado um cronômetro digital DCS -368 (Profissional Quartz Timer) para medir o TMF; um gravador Panasonic RQ-L11 e fita cassete TDK-60 minutos para arquivo e confirmação do TMF medido e o aparelho PFE míni-Wrigth para a medição do sopro expiratório.

A análise dos resultados foi obtida através de gráficos comparativos entre a população pesquisada e a literatura.

O presente estudo foi aprovado pelo Comitê de Ética em Pesquisa da Fundação Hospitalar do Estado de Minas Gerais (CEP-FHEMIG), sob o número 230/2004 que o considerou sem risco e com necessidade de consentimento livre e esclarecido.

Foi utilizado cálculo simples para obtenção de médias. Desta forma foram calculadas as médias de cada uma das emissões do TMF, assim como a média do PFE, em relação a cada grupo de gravidade da asma. As médias obtidas do TMF foram comparadas com a média obtida do PFE. Não foi possível aplicar teste estatístico devido ao número grande de variáveis e reduzido número populacional de asmáticos pesquisado.

\section{Protocolo de Avaliação de Pesquisa}

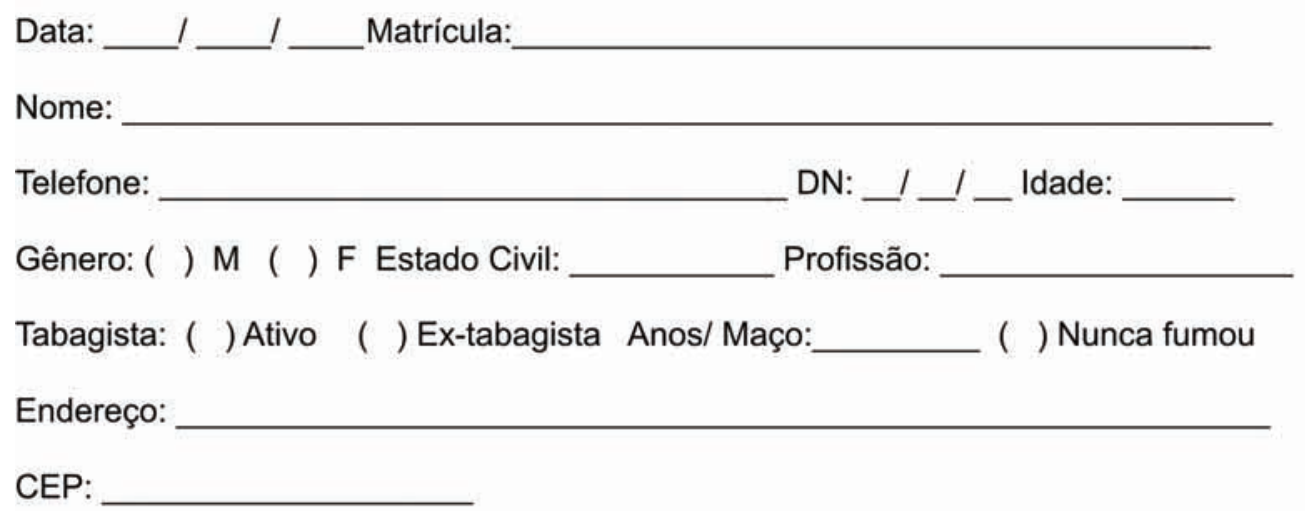

\section{I-EMISSÃO DOS SONS DA FALA: TMF}

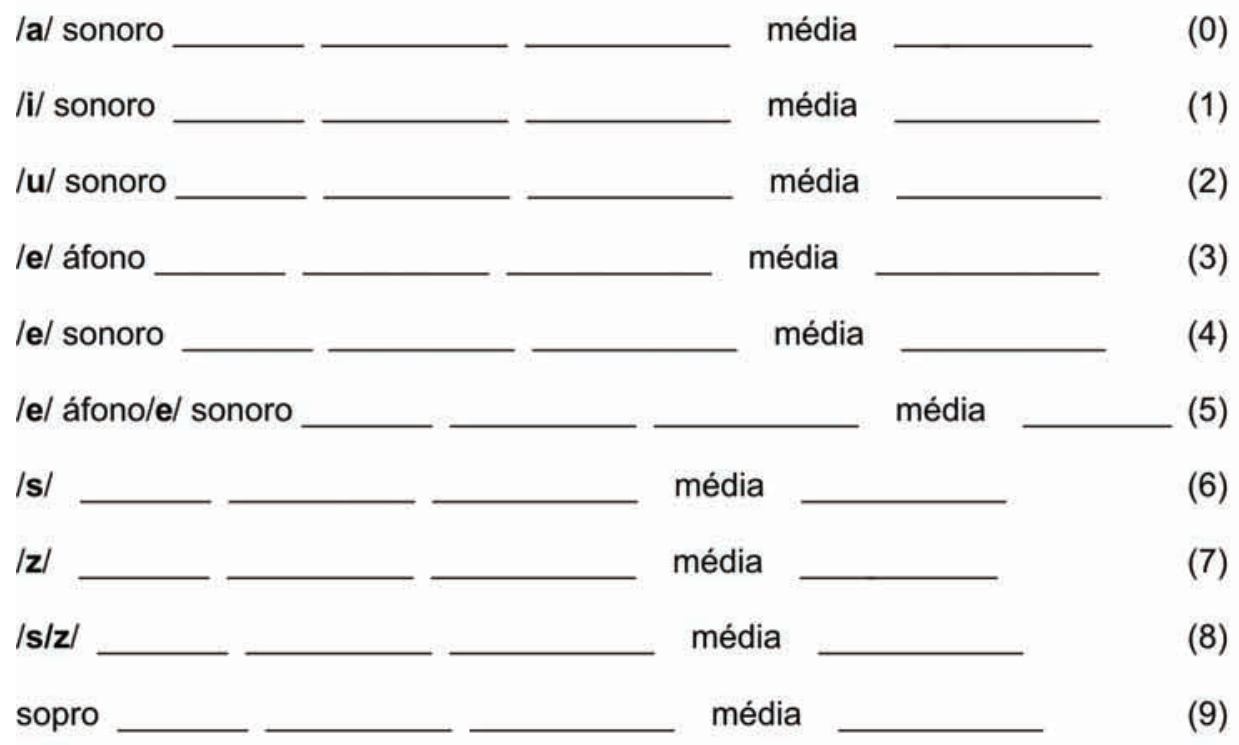




\section{II-ESCORE DE SINTOMAS (baseado no consenso de pacientes asmáticos)}

\section{S. Diurno: $0 \quad 1 \quad 2 \quad 3 \quad 4$}

0 - sem sintoma de asma (sem falta de ar, chieira), atividades normais, consegue fazer esporte;

1 - sintomas leves, sem prejudicar as atividades normais. Dificuldades para exercicios ou correr;

2 - sintomas moderados chegando a prejudicar uma ou maisatividades normais;

\section{RESULTADOS}

Os dados obtidos na amostra dos 16 pacientes do ambulatório de pneumologia do HJK, todos com asma pura sem outra patologia associada, revelaram que de acordo com a classificação da asma havia $12,50 \%$ (2) com asma tratada; $18,75 \%$ (3) com asma persistente leve $43,75 \%$ (7) com asma persistente moderada $6,25 \%$ (1) com asma moderada grave e $18,75 \%$ (3) com asma persistente grave.

O estudo da média das emissões encontrada para os valores do Tempo Máximo de Fonação (TMF) em relação ao grau da asma mostrou que na asma trata- da houve valores de normalidade, os sujeitos com a asma persistente leve, persistente moderada, moderada grave e persistente grave foram encontrados valores reduzidos de TMF (Figura 1).

Pelo fato de o Pico de Fluxo Expiratório (PFE) apresentar variabilidade em relação ao valor de sua medida, mesmo que em um único sujeito, tornou-se difícil obter uma medição confiável para se estudar a possível relação do PFE e o TMF. Pôde-se observar que quando o TMF encontrava-se reduzido ocorria também uma redução do PFE e quando havia um aumento do TMF era encontrado no PFE valor também maior, o que poderia sugerir uma relação direta entre essas medidas (Figuras 1 a 4).

\begin{tabular}{|c|c|c|c|c|c|c|c|c|c|c|c|c|c|}
\hline $\begin{array}{l}\text { Grau } \\
\text { Asma }\end{array}$ & $\%$ & $\begin{array}{l}\text { Total } \\
\text { Pac. }\end{array}$ & \begin{tabular}{|l|} 
média \\
/a/seg \\
\end{tabular} & \begin{tabular}{|l|} 
média \\
li/seg \\
\end{tabular} & \begin{tabular}{|l|} 
média \\
/u/seg
\end{tabular} & \begin{tabular}{|c|} 
média \\
leaf/
\end{tabular} & $\begin{array}{l}\text { média } \\
\text { lesn/ }\end{array}$ & \begin{tabular}{|c|} 
média \\
eaf/eson
\end{tabular} & \begin{tabular}{|l|} 
média \\
/s/seg
\end{tabular} & \begin{tabular}{|l|} 
média \\
/z/seg \\
\end{tabular} & \begin{tabular}{|c|} 
média \\
$\mathrm{s} / \mathrm{z}$ \\
\end{tabular} & \begin{tabular}{|l|} 
média \\
Sopro \\
\end{tabular} & \begin{tabular}{|c} 
média \\
$\mathrm{PFE}$ \\
$\mathrm{I} / \mathrm{s}$
\end{tabular} \\
\hline 1 & 12,50 & 2 & 20,2 & 27,24 & 24,84 & 14,08 & 22,98 & 0,61 & 12,36 & 15,99 & 0,76 & 15,34 & 8,83 \\
\hline 2 & 18,75 & 3 & 8,72 & 9,29 & 11,14 & 7,53 & 10,56 & 0,72 & 8,6 & 7,42 & 1,14 & 8,3 & 7,19 \\
\hline 3 & 43,75 & 7 & 4,67 & 5,11 & 5,58 & 6,3 & 6,38 & 1,16 & 6,1 & 6,57 & 1 & 5,2 & 4,2 \\
\hline 4 & 6,25 & 1 & 4,35 & 2,91 & 2,6 & 3,93 & 3,27 & 1,2 & 6,51 & 4,05 & 1,6 & 4,38 & 3,91 \\
\hline 5 & 18,75 & 3 & 7,4 & 9,03 & 8,82 & 7,31 & 8,81 & 0,83 & 7,89 & 8,48 & 0,93 & 6,07 & 7,16 \\
\hline
\end{tabular}

PFE = Pico de Fluxo Expiratório; /eaf/ = /e/ áfono; /asn/ = /e/ sonoro seg = segundos; $1 / \mathrm{s}=$ litros por segundo Grau da asma: $1=$ Tratada 2 = Leve 3 =Moderada 4 =Moderada/Grave $5=$ Grave

Figura 1 - Quadro geral dos valores encontrados no estudo

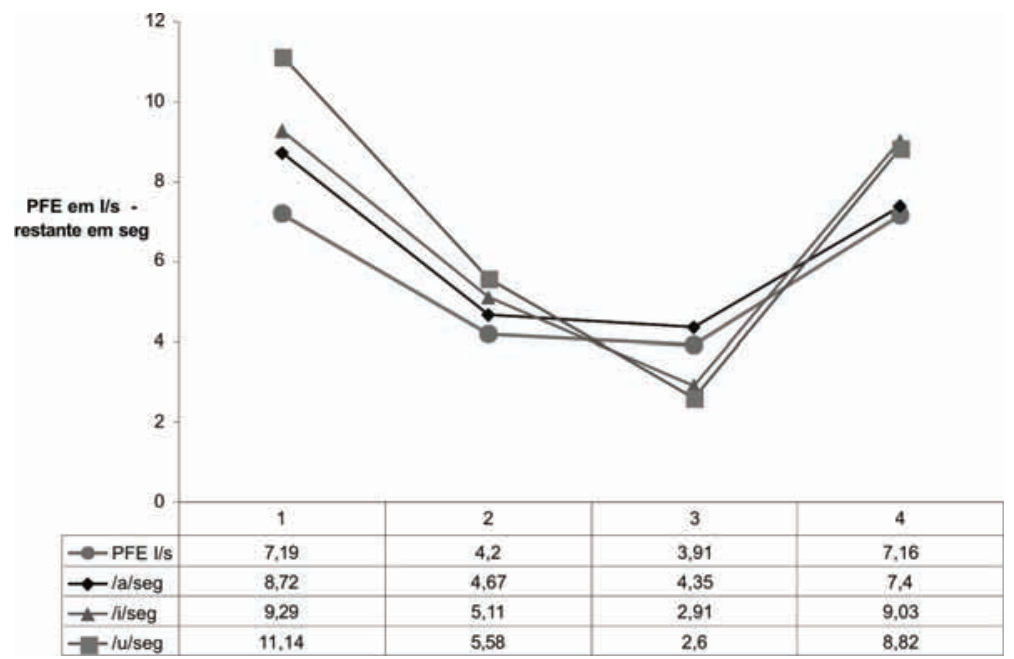

PFE = Pico de Fluxo Expiratório; seg = segundos; TMF = Tempo Máximo de Fonação l/s = litros por segundo Asma: $1=$ tratada $2=$ leve $3=$ moderada $4=$ moderada/grave $5=$ grave

Figura 2 - Relação entre o PFE e TMF da emissão prolongada do /a/, /i/, /u/, levando em consideração a classificação da asma 
514 Rossi DC, Munhoz DF, Nogueira CR, Oliveira TCM, Britto ATBO

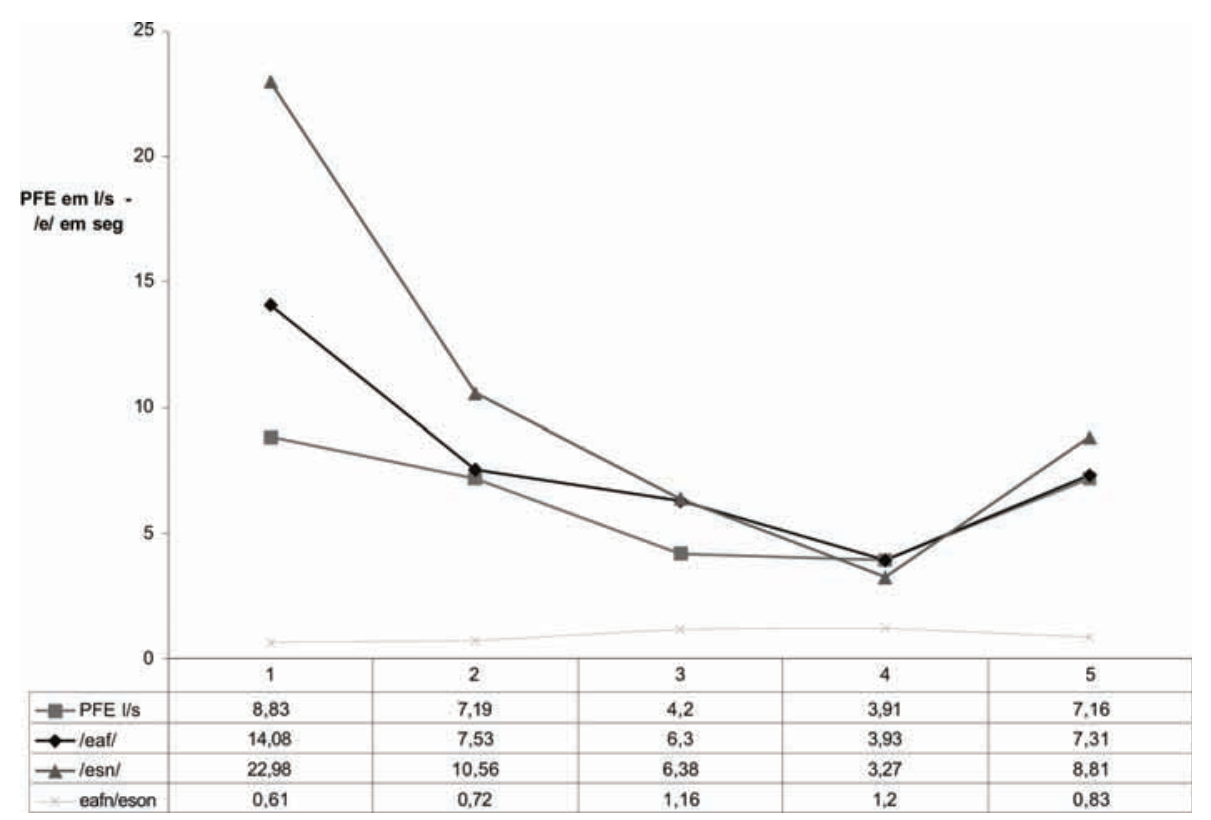

PFE = Pico de Fluxo Espiatório; l/s = litros por segundo; /eaf/ = /e/ áfono; /esn/ = /e/ sonoro; seg = segundos Asma: $1=$ tratada $2=$ leve $3=$ moderada $4=$ moderada/grave $5=$ grave

Figura 3 - Relação entre o PFE e TMF do /eafn/, /eson/, eafn/eson, em pacientes asmáticos

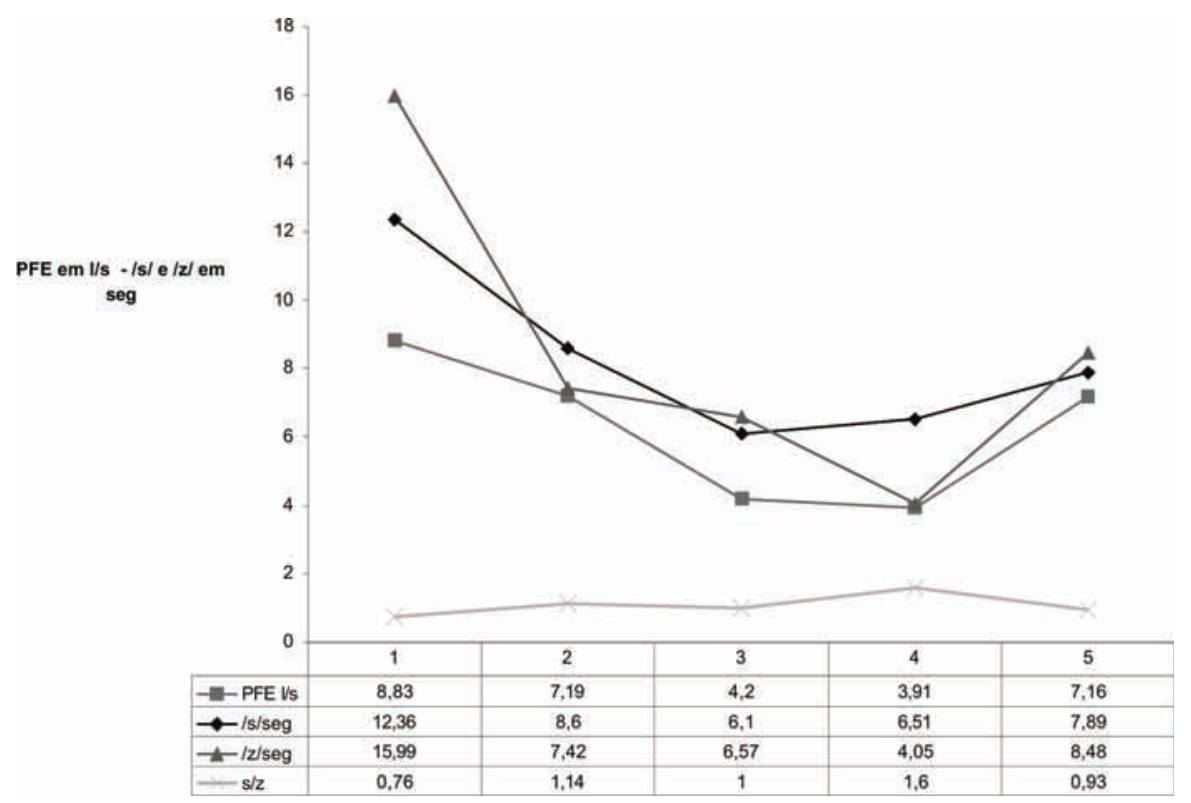

PFE = Pico de Fluxo Espiatório; seg = segundos; TMF = Tempo Máximo de Fonação; $1 / s$ = litros por segundo Asma: $1=$ tratada $2=$ leve $3=$ moderada $4=$ moderada/grave $5=$ grave

Figura 4 - Relação entre o PFE e as médias doTMF para /s/, /z/, s/z, em pacientes asmáticos 


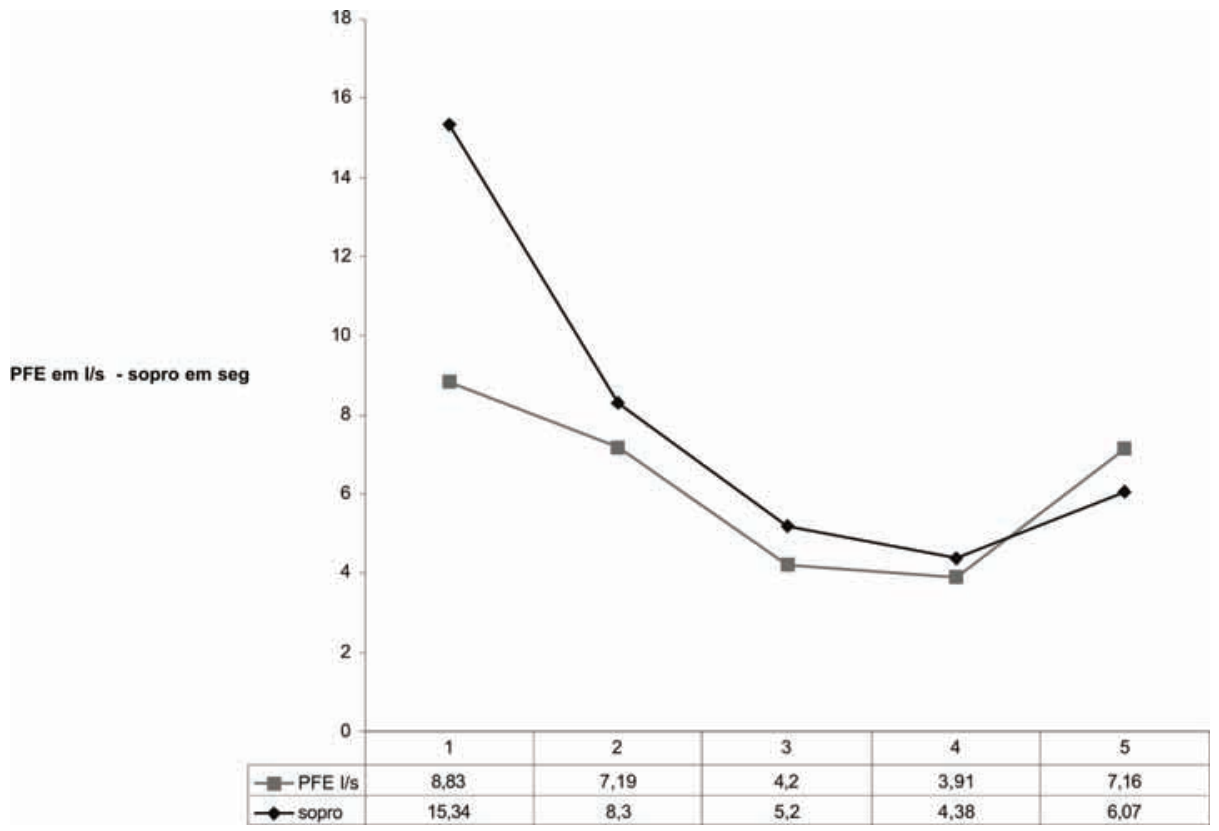

$\mathrm{PFE}=$ Pico de Fluxo Espiatório; $1 / \mathrm{s}=$ litros por segundo; seg = segundos

Asma: $1=$ tratada $2=$ leve $3=$ moderada $4=$ moderada/grave $5=$ grave

Figura 5 - Relação entre o PFE e o sopro em pacientes asmáticos

\section{DISCUSSÃO}

Todos os indivíduos que participaram do estudo apresentaram respiração do tipo costal superior, rigidez e tensão cervical, além de variações na voz (tensa, soprosa e com instabilidade fonatória) ${ }^{18}$. Um motivo para acreditar que variáveis na respiração podem influenciar o TMF é que a laringe se une mecanicamente ao aparelho respiratório, permitindo que ela sofra força direta deste último ${ }^{19}$. Para se obter um volume pulmonar alto o diafragma se deprime puxando a traquéia e a laringe caudalmente, tendendo a abduzir as pregas vocais ${ }^{20}$. O volume pulmonar afeta a fonte glótica. Durante a inspiração a glote se alarga pelo afastamento das cartilagens aritenóides devido a uma tração da traquéia resultante da expansão pulmonar ${ }^{21}$. Esse afastamento das aritenóides resulta na abdução das pregas vocais. Através do estudo dos movimentos brônquicos, utilizando raios $\mathrm{x}, \mathrm{a}$ carina sofre um deslocamento inferior em média de $21 \mathrm{~mm}$ durante a inspiração ${ }^{22}$.

Nos sujeitos diagnosticados com asma persistente leve o valor do TMF ficou entre 9 e 11 segundos; na asma persistente moderada permaneceu entre 4 e 6 segundos; na asma moderada grave foram encontrados valores de TMF entre 2 e 4 segundos e, na asma persistente grave, o TMF ficou entre 7 e 9 segundos. Nos sujeitos com asma tratada foram encontrados valores no TMF para os fonemas /a/, /i/, /u/ e /e/ sonoro, valores acima da média esperada. Nos sujeitos considerados normais, os valores variavam entre 20 e 27 segundos.
Isso evidencia que o TMF deve ser melhor pesquisado e que deve ser levado em consideração as variáveis tais como idade (o próprio enveIhecimento leva a diminuição do fluxo pulmonar), gênero (diferença da força muscular entre homem e mulher), altura (diferença anatômica do aporte torácico e conseqüentemente do volume pulmonar) e também quanto as compensações individuais.

Contrariamente ao que ocorre em indivíduos adultos normais, nos sujeitos asmáticos não foram encontrados valores maiores do TMF para o gênero masculino. $4,8-10,23$.

Todos os sujeitos fizeram uso do ar de reserva.

O TMF e o PFE são influenciados por diversos fatores que interagem entre si simultaneamente de uma forma complexa e individual, existindo também o fator subjetivo do examinador. De acordo com as estratégias de compensação laríngeas utilizadas das formas mais variadas, o TMF não seria adequado para uma avaliação generalizada da população. Como essas compensações são características individuais, o TMF poderia ser considerado um bom parâmetro de acompanhamento individual.

Os asmáticos têm a capacidade de compensar temporariamente uma maior necessidade para a fala, com prejuízo à necessidade metabólica, sob sacrifício, resultando posteriormente em respiração curta, hiperventilação e até necessidade de respiração gasosa (oxigênio). Seu estudo demonstrou que houve uma redução geral na CV e volume expiratório forçado em litros por segundo $(\mathrm{L} / \mathrm{s})$ e isso pode afetar a fala por mecanismos metabólicos (alcalose respiratória), aerodinâmicos 
(resistência ao fluxo aéreo pulmonar) ou biomecânicos (cinética da parede torácica) ${ }^{11}$.

Portanto, o estudo de pacientes com insuficiência respiratória deve ser baseado em um entendimento do mecanismo ventilatório e suas anormalidades. Os fundamentos mecânicos em outras doenças respiratórias são diferentes do que ocorre na patologia da asma e devem ser estudadas por mecanismos diferentes ${ }^{13}$.

\section{CONCLUSÃO}

Foi possível observar uma proporcionalidade entre as medidas TMF e PFE de forma concomitante. Embora, não se pode afirmar que há uma relação direta entre as medidas pela não aplicabilidade de teste estatístico devido ao limitado número populacional da pesquisa e grande número de variáveis.

\begin{abstract}
Purpose: to investigate the possible relationship between Expiratory Flow Peak (EFP) and Maximum Phonation Duration (PMD) for asthmatic patients Methods: the research focused on 16 individuals diagnosed with pure asthma, where 9 were female and 7 were males, treated at the pneumology clinical services of Júlia Kubitschek Hospital. The pneumology team diagnosed, classified the asthma and performed the EFP test. The patients were submitted afterwards to PMD speech therapy evaluation. Results: within the treated patients there were $12.50 \%$ (2) with treated asthma, $18.75 \%$ (3) with light persistent asthma, $43.75 \%$ (7) with moderate persistent asthma, $6.25 \%$ (1) with severe moderate asthma and $18.75 \%$ (3) with severe persistent asthma. It was found that when PMD had decreased there was a decrease in EFP as well, and that when there was an increase of PMD the EFP value would also increase. Conclusion: it was possible to note a relation and a correlation between PMD and EFP measures, suggesting a direct relation between these measurements.
\end{abstract}

KEYWORDS: Peak Expiratory Flow Rate; Asthma; Speech, Language and Hearing Sciences; Pulmonary Disease (Speciality)

\section{REFERÊNCIAS}

1.Ramig LO, Verdolini K. Treatment efficacy: voice disorders. J Speech Lang Hear Res. 1998; 41(1):S101-16.

2.Boone DR, McFarlane SC. A voz e a terapia vocal. 5. ed. Porto Alegre: Artes Médicas; 1994.

3.'T Hart J, et al. A perceptual study of intonation. New York: Cambridge University Press; 1990. p. 10-7.

4.Pinho SMR. Fundamentos em fonoaudiologia: tratando os distúrbios da voz. Rio de Janeiro: Guanabara Koogan; 2003. p. 24-9.

5.Solomon NP, Garlitz SJ, Milbrath RL. Respiratory and laryngeal contributions to maximum phonation duration. J Voice. 2000; 14(3):331-40.

6. Diretrizes para testes de função pulmonar. J Pneumol. 2002; 28(3):2-82.

7. Yanagihara N, Koike Y. The regulation of sustained phonation. Folia Phoniatr. 1967; 19(1):1-18.

8.Behlau M, Pontes P. Avaliação e tratamento das disfonias. São Paulo: Lovise; 1995.

9. Behlau M, Rodrigues S, Azevedo R, Gonçalves MI, Pontes P.Avaliação e terapia de voz. In: Lopes Filho O. Tratado de fonoaudiologia. São Paulo: Roca; 1997. p. 607-58.

10. Behlau M. A voz do especialista. v. 1. Rio de Janeiro: Revinter; 2001.
11. Lee L, Chamberlain LG, Loudon RG, Stemple JC. Speech segment durations produced by healthy and asthmatic subjects. J Speech Hear Disord. 1988; 53(2):186-93.

12. III Consenso Brasileiro no Manejo da Asma. J Pneumol. 2002; 28(1):s1-51.

13. Loudon RG, Lee L, Holcomb BJ. Volumes and breathing patterns during speech in healthy and asthmatic subjects. J Speech Hear Res. 1988; 31(2):219-27.

14. Silva LCC, Rubin AS, Silva LMC. Avaliação funcional pulmonar. Rio de Janeiro: Revinter; 2000.

15. Dias RM et al. Testes de função respiratória: do laboratório à aplicação clínica com 100 exercícios para diagnóstico. São Paulo: Atheneu; 2000.

16. Tanaka S, Hirano M, Chijiwa K. Some aspects of vocal fold bowing. Ann Otol Rhinol Laryngol. 1994; 103(5):357-62.

17. Behlau M. O melhor que vi e ouvi III: atualização em laringe e voz. Rio de Janeiro: Revinter; 2001.

18. Colton RH, Casper JK. Compreendendo os problemas de voz. Porto Alegre: Artes Médicas; 1996.

19. Dângelo JG, Fattini CA. Anatomia humana sistêmica e segmentar: para estudante de medicina. 2. ed. São Paulo: Atheneu; 1998.

20. Hoit JD, Solomon NP, Hixon TJ. Effect of lung 
volume on voice onset time (VOT). J Speech Hear Res. 1993; 36(3):516-20.

21. Iwarsson J, Thomasson M, Sundberg J. Effects of lung volume on the glottal voice source. $J$ Voice. 1998; 12(4):424-33.
22. Maklin C. X ray studies on brochial movements. An J Anat. 1925; 35:303-20.

23. Costa HO, Silva MAA. Voz cantada: evolução, avaliação e terapia fonoaudiológica. São Paulo: Lovise; 1998.

Recebido em: 25/04/2006

Aceito em: 22/09/2006

\section{Endereço para correspondência}

Av. Professor Mário Werneck, 2444/904

Belo Horizonte - MG

CEP: $30575-180$

Tel: (31) 33770313 / (31) 32416826

E-mail:deborarossi@terra.com.br 